Supporting Information

\title{
Novel Mechanistic Insights into Methane Activation over Fe and Cu Active Sites in Zeolites: A Comparative DFT Study Using Meta-GGA Functionals
}

Muhammad Haris Mahyuddin, ${ }^{* a, b}$ Aleksandar Staykov, ${ }^{c}$ Adhitya Gandaryus Saputro, a,b Mohammad Kemal Agusta, ${ }^{a, b}$ Hermawan Kresno Dipojono, ${ }^{* a, b}$ and Kazunari Yoshizawa*,a,d

a Research Group of Advanced Functional Materials, Faculty of Industrial Technology, Institut Teknologi Bandung, Jl. Ganesha 10 Bandung 40132, Indonesia

${ }^{b}$ Research Center for Nanoscience and Nanotechnology, Institut Teknologi Bandung, Jl. Ganesha 10 Bandung 40132, Indonesia

c International Institute for Carbon-Neutral Energy Research, Kyushu University, Fukuoka 819-0395, Japan

${ }^{d}$ Institute for Materials Chemistry and Engineering and IRCCS, Kyushu University, Fukuoka 819-0395, Japan

* To whom all correspondence should be addressed

(e-mail: haris[at]tf.itb.ac.id, dipojono[at]tf.itb.ac.id, and kazunari[at]ms.ifoc.kyushu-u.ac.jp). 


\section{Table of Contents:}

Figure S1 Complete energy diagrams of methane activation over $[\mathrm{FeO}]^{2+}-\mathrm{MFI}$ in the quintet and triplet states.

Figure S2 Projected density of states (PDOS) for RC of [FeO] ${ }^{2+}$ MFI in the quintet state, calculated by the TPSS-D2 and MSO-D2 functionals.

Figure S3 Complete energy diagrams of methane activation over $\left[\mathrm{Cu}_{2}(\mu-\mathrm{O})\right]^{2+}-\mathrm{MFI}$ in the triplet and open-shell singlet states.

Figure S4 Projected density of states (PDOS) for RC and RI of $\left[\mathrm{Cu}_{2}(\mu-\mathrm{O})\right]^{2+}-\mathrm{MFI}$ in the triplet state, calculated by the MS2-D2 and SCAN-D2 functionals.

Figure S5 Complete energy diagrams of methane activation over $\left[\mathrm{Cu}_{3}(\mu-\mathrm{O})_{3}\right]^{2+}-\mathrm{MOR}$ in the quartet and doublet states.

Figure S6 Projected density of states (PDOS) for $\mathbf{R C}$ and $\mathbf{R I}$ of $\left[\mathrm{Cu}_{3}(\mu-\mathrm{O})_{3}\right]^{2+}-\mathrm{MOR}$ in the quartet state, calculated by the MS2-D2 and SCAN-D2 functionals.

Table S1 Comparison of $\mathrm{CH}_{4}$ Adsorption Energies Calculated with and without the D2 or D3 Method of Dispersion Correction

Figure S7 Visualization of atomic spin densities and $\mathrm{C}-\mathrm{H}$ bond length for $\mathbf{R C}$, TS, and $\mathbf{R I}$ of $[\mathrm{FeO}]^{2+}$ ZSM-5.

Figure S8 Visualization of atomic spin densities and $\mathrm{C}-\mathrm{H}$ bond length for $\mathbf{R C}$, TS, and $\mathbf{R I}$ of $\left[\mathrm{Cu}_{3}(\mu-\right.$ O) 3$]^{2+}-Z S M-5$.

Figure S9 Visualization of atomic spin densities and $\mathrm{C}-\mathrm{H}$ bond length for $\mathbf{R C}$, TS, and $\mathbf{R I}$ of $\left[\mathrm{Cu}_{3}(\mu-\right.$ O) $\left.]_{3}\right]^{2+-M O R}$. 
(a) TPSS-D2

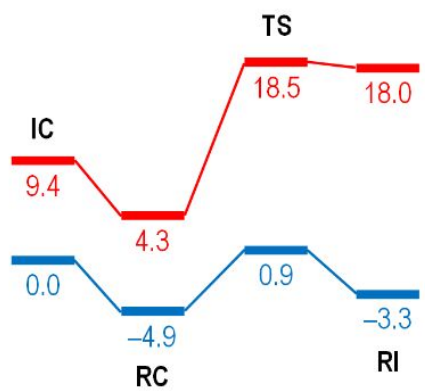

(d) MS1-D2

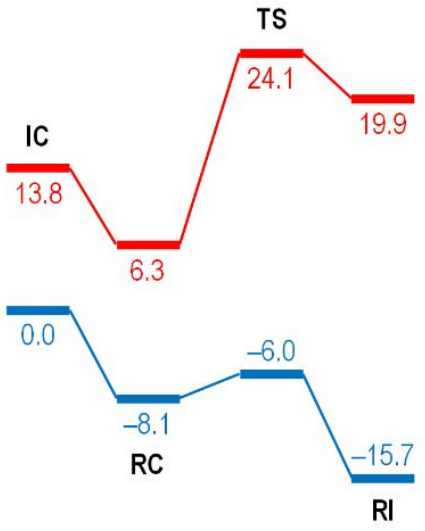

(b) RTPSS-D2

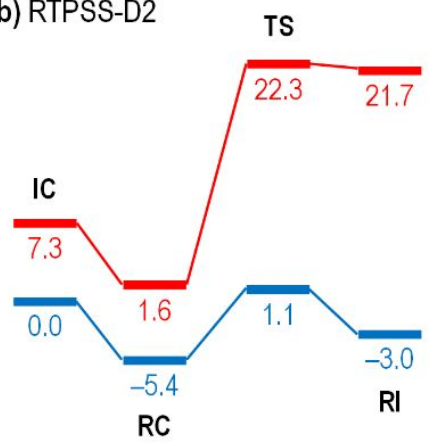

(e) MS2-D2

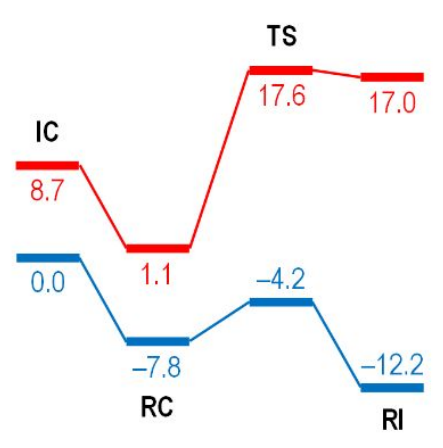

(c) MSO-D2

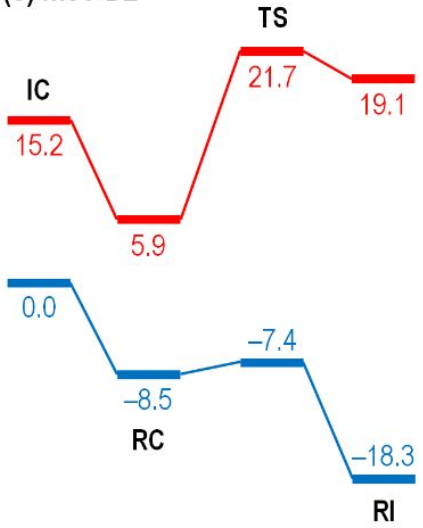

(f) SCAN-D2
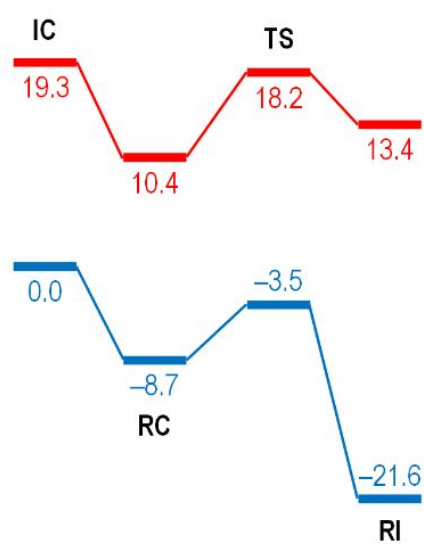

Figure S1. Complete energy diagrams of methane activation over $[\mathrm{FeO}]^{2+}-\mathrm{MFI}$ in the quintet (blue lines) and triplet (red lines) states. Energies are in $\mathrm{kcal} / \mathrm{mol}$. 


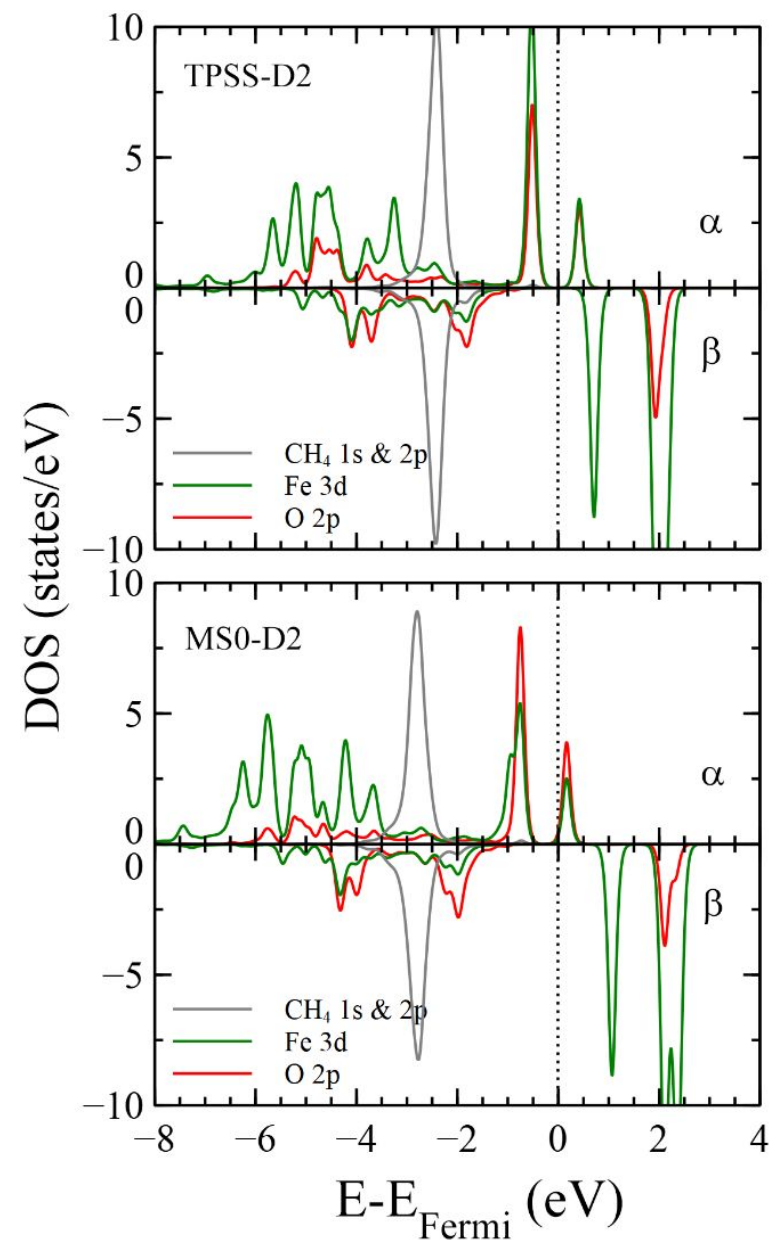

Figure S2. Projected density of states (PDOS) for RC of $[\mathrm{FeO}]^{2+-} \mathrm{MFI}$ in the quintet state, calculated by the TPSS-D2 (top) and MS0-D2 (bottom) functionals.

- Both of the TPSS-D2 and SCAN-D2 functionals show the presence of an unoccupied Fe-d orbital both in the $\alpha$ and $\beta$ spins near the Fermi level (at about $0.2 \sim 0.5$ and $0.7 \sim 1.0 \mathrm{eV}$, respectively for the $\alpha$ and $\beta$ spins), which indicates the characteristic of $\mathrm{Fe}^{\mathrm{IV}}$ electronic structure.

- The $\alpha$-LUMO (i.e. the Fe-O $\sigma^{*}$ orbital) predicted by the MSO-D2 functional is lower in energy than that predicted by the TPSS-D2 functional, thus causing the $[\mathrm{FeO}]^{2+}$ predicted by the MS0-D2 functional to be more reactive toward methane. 
(a) TPSS-D2

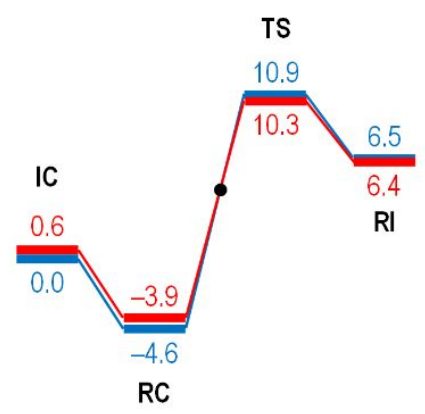

(d) MS1-D2

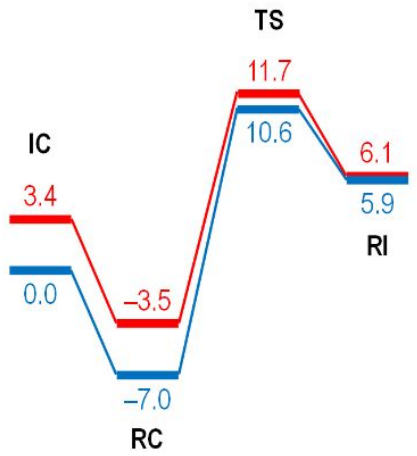

(b) RTPSS-D2

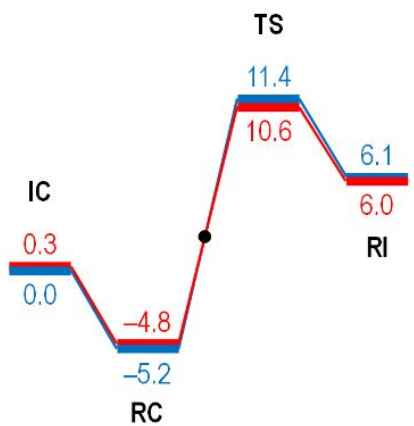

(e) MS2-D2

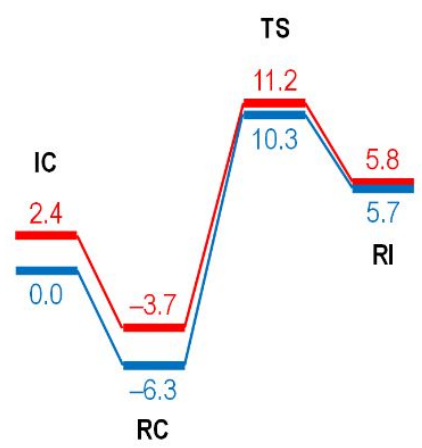

(c) MSO-D2

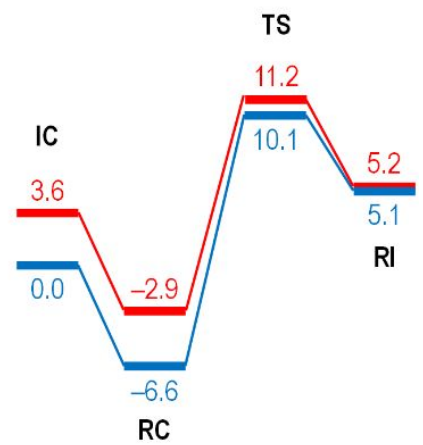

(f) SCAN-D2

Figure S3. Complete energy diagrams of methane activation over $\left[\mathrm{Cu}_{2}(\mu-\mathrm{O})\right]^{2+}-\mathrm{MFI}$ in the triplet (blue lines) and open-shell singlet (red lines) states. Energies are in $\mathrm{kcal} / \mathrm{mol}$. 
RC
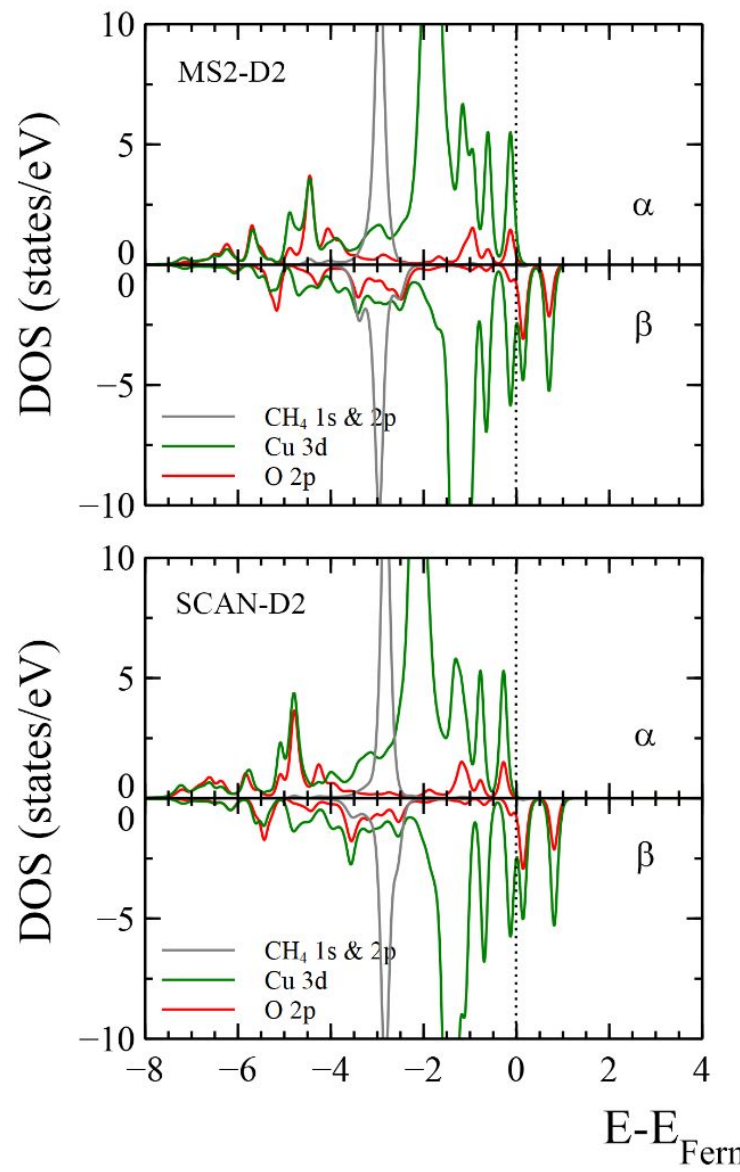

RI
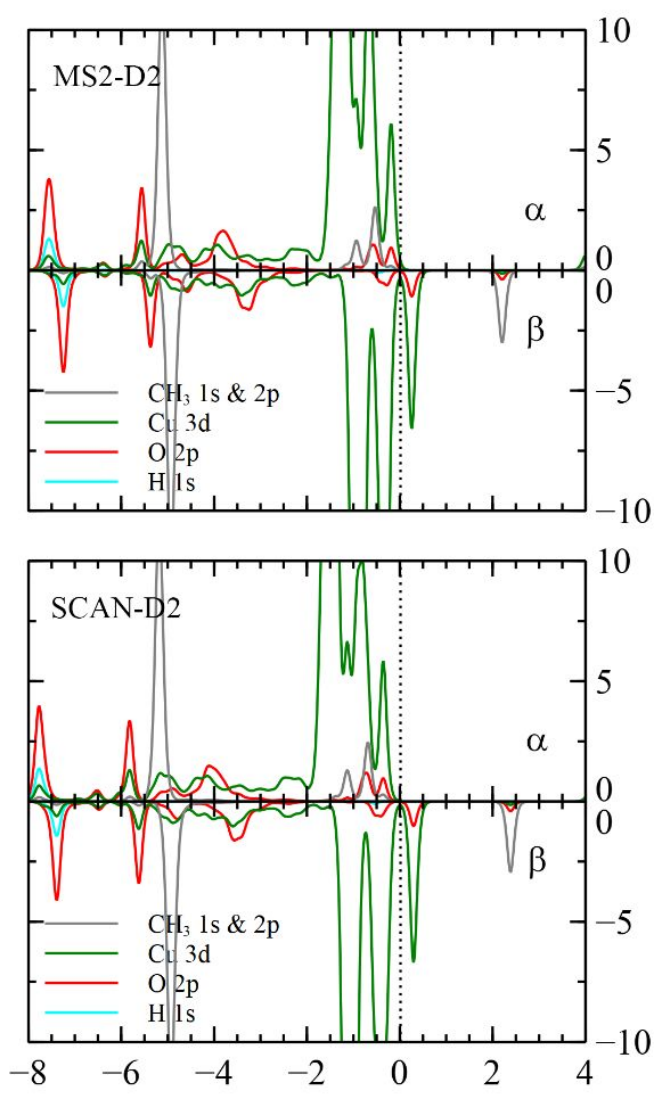

$(\mathrm{eV})$

Figure S4. Projected density of states (PDOS) for $\mathbf{R C}$ and $\mathbf{R I}$ of $\left[\mathrm{Cu}_{2}(\mu-\mathrm{O})\right]^{2+}-\mathrm{MFI}$ in the triplet state, calculated by the MS2-D2 and SCAN-D2 functionals.

- In RC, both of the MS2-D2 and SCAN-D2 functionals show the presence of two occupied Cu-d orbitals ( $\alpha$ spin) at about -0.4 and $-0.7 \mathrm{eV}$ and two unoccupied Cu-d orbitals ( $\beta$ spin) at about 0.2 and $0.7 \mathrm{eV}$. This indicates two singly occupied molecular orbitals (SOMOs), which characterize the involvement of two $\mathrm{Cu}^{2+}$ ions in the active species.

- A slight stabilization of the whole $\alpha$ orbitals is observed when comparing the PDOSs of RC calculated by the SCAN-D2 and MS2-D2 functionals. As a result, the $\alpha$ electron of ${ }^{\circ} \mathrm{CH}_{3}$ in RI calculated by the SCAN-D2 functional is slightly more stable. 
(a) TPSS-D2

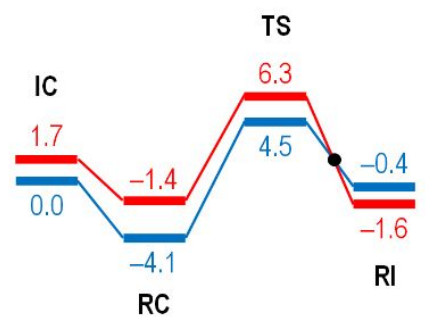

(d) MS1-D2

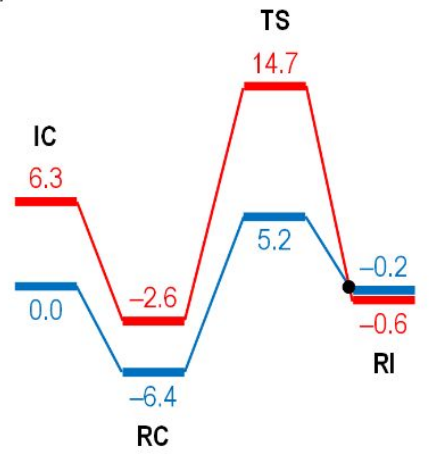

(b) RTPSS-D2

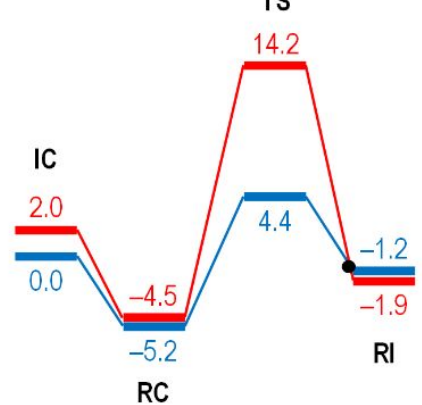

(e) MS2-D2

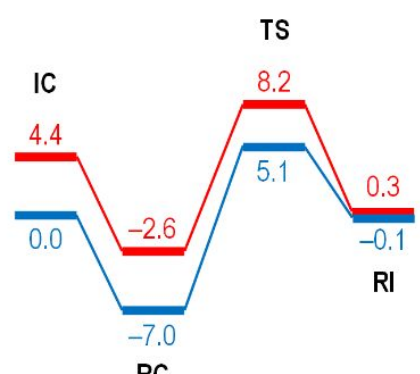

$\mathrm{RC}$ (c) MSO-D2

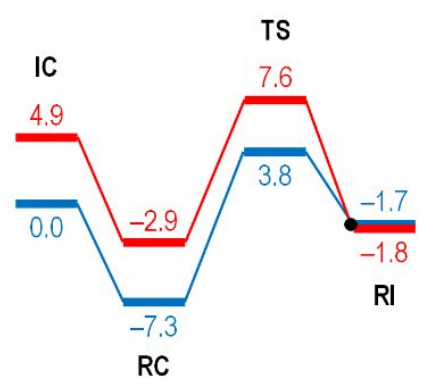

(f) SCAN-D2

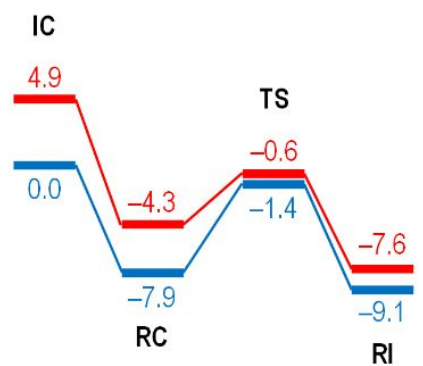

Figure S5. Complete energy diagrams of methane activation over $\left[\mathrm{Cu}_{3}(\mu-\mathrm{O})_{3}\right]^{2+}-\mathrm{MOR}$ in the quartet (blue lines) and doublet (red lines) states. Energies are in $\mathrm{kcal} / \mathrm{mol}$. 
RC
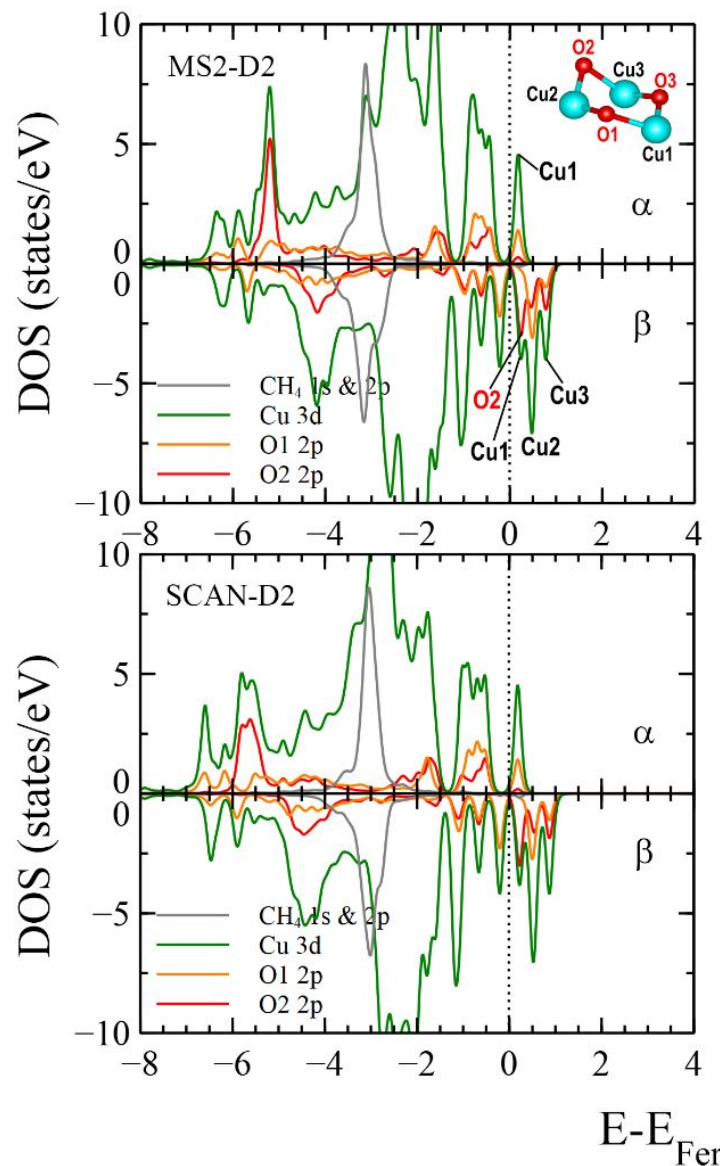

RI
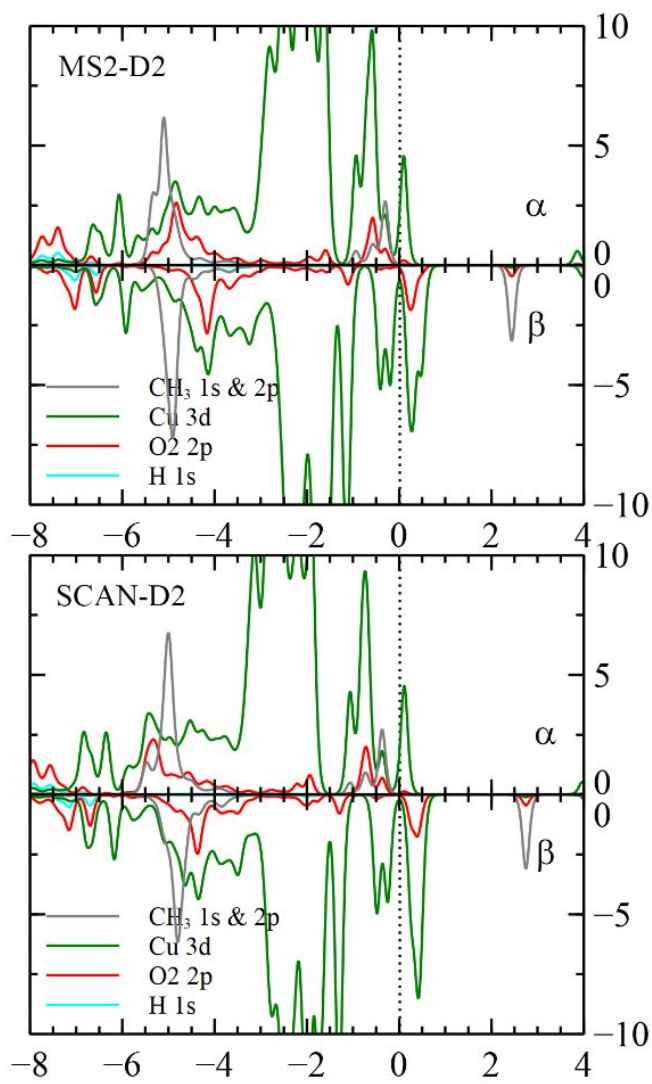

$(\mathrm{eV})$

Figure S6. Projected density of states (PDOS) for $\mathbf{R C}$ and $\mathbf{R I}$ of $\left[\mathrm{Cu}_{3}(\mu-\mathrm{O})_{3}\right]^{2+}-\mathrm{MOR}$ in the quartet state, calculated by the MS2-D2 and SCAN-D2 functionals.

- In RC, both of the MS2-D2 and SCAN-D2 functionals show a pair of unoccupied Cu1-d orbitals in the $\alpha$ and $\beta$ spins near the Fermi level (at about 0.3 and $0.6 \mathrm{eV}$, respectively for the $\alpha$ and $\beta$ spins), indicating a $\mathrm{Cu}^{3+}$ ion, which is different from $\mathrm{Cu}^{+}$ion that would result in all occupied $\mathrm{Cu}-\mathrm{d}$ orbitals. At the same time, the presence of two unoccupied Cu2-d and Cu3-d orbitals as well as one unoccupied O2-p orbital in the $\beta$ spin near the Fermi level indicates two $\mathrm{Cu}^{2+}$ ions and one $\mathrm{O}^{\cdot}$ radical. The Cu1-d and O2-p orbitals in the $\beta$ spin are not hybridized with each other since the $\mathrm{Cu} 1$ and $\mathrm{O} 2$ atoms are located away from each other.

- The presence of unoccupied O1-p orbital in the $\beta$ spin is due to a hybridization between Cu2 and $\mathrm{O} 1$ atoms. The same description also applies for $\mathrm{O} 3$ atom (i.e. Cu3-d and O3-p hybridization).

- In RI, there seems only one unoccupied Cu-d orbital in the $\beta$ spin. However, there are actually three unoccupied $\mathrm{Cu}-\mathrm{d}$ orbitals, which are energetically very close to each other. 
Table S1. Comparison of $\mathrm{CH}_{4}$ Adsorption Energies $\left(E_{a d s}\right)$ on $[\mathrm{FeO}]^{2+}-\mathrm{ZSM}-5$ Calculated with and without the D2 or D3 Method of Dispersion Correction.

\begin{tabular}{lcccccc}
\hline & RTPSS & RTPSS-D2 & RTPSS-D3 & MS0 & MS0-D2 & MS0-D3 \\
\hline$E_{\text {ads }}(\mathrm{kcal} / \mathrm{mol})$ & 0.4 & -5.4 & -6.6 & -2.4 & -8.5 & -9.3 \\
\hline
\end{tabular}

- As shown in this table, dispersion correction is essential for correctly predicting the $\mathrm{CH}_{4}$ adsorption energy inside the microporous of zoolites. This suggests that dispersion correction still should be included even for meta-GGA functionals.

- This table also shows that even without the dispersion correction, the MSO functional already predicts a negative value of $E_{a d s}$ (exothermic), which is in contrast to $E_{\text {ads }}$ predicted by the RTPSS functional. This indicates how weak interactions except the van der Waals dispersive interactions are well treated by the MS functionals. 

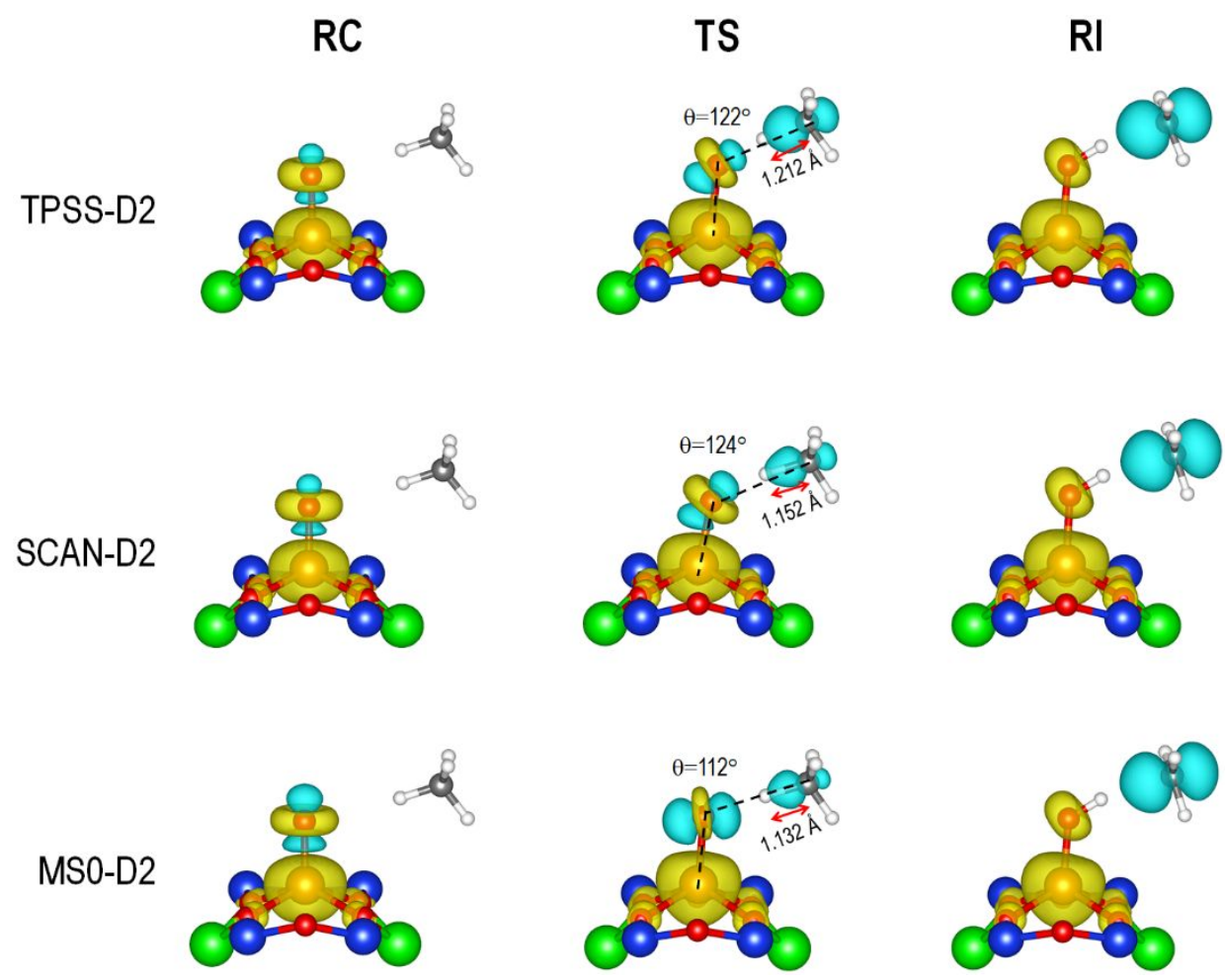

Figure S7. Visualization of atomic spin densities (yellow and blue isosurfaces correspond to positive and negative spin densities, respectively) and $\mathbf{C}-\mathbf{H}$ bond length for $\mathbf{R C}, \mathbf{T S}$, and $\mathbf{R I}$ of $[\mathrm{FeO}]^{2+}-\mathrm{ZSM}-5$ in the corresponding ground state. 


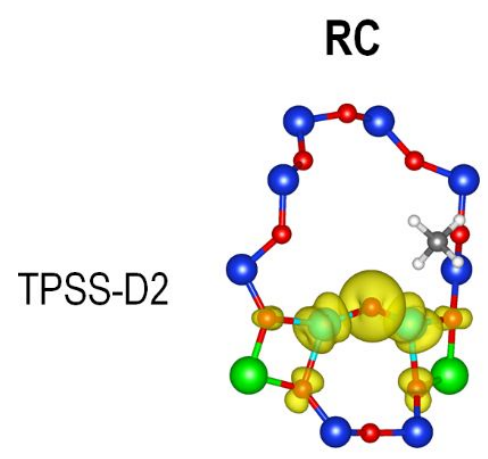

TS
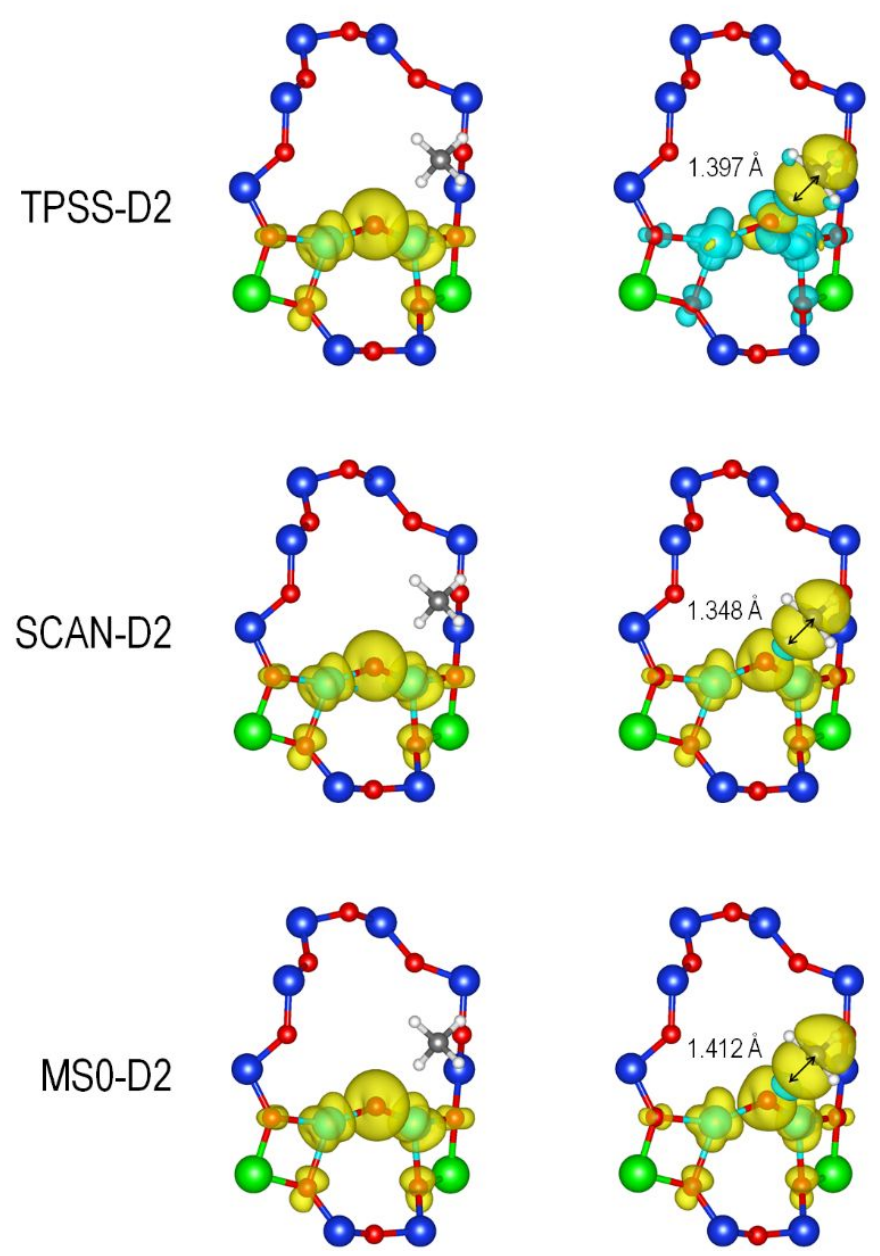
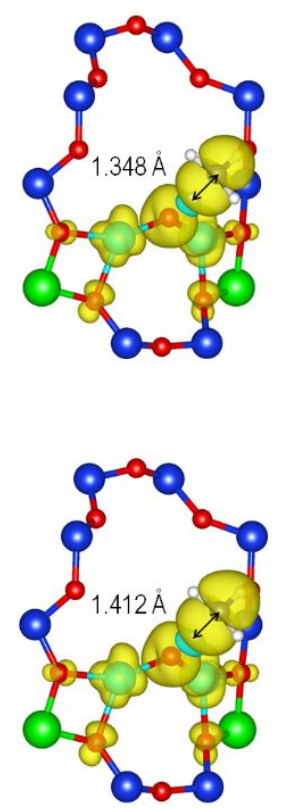

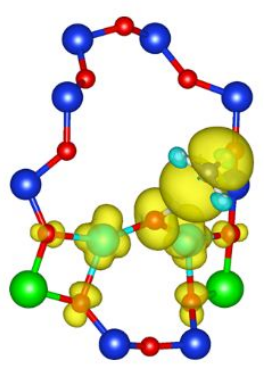

RI
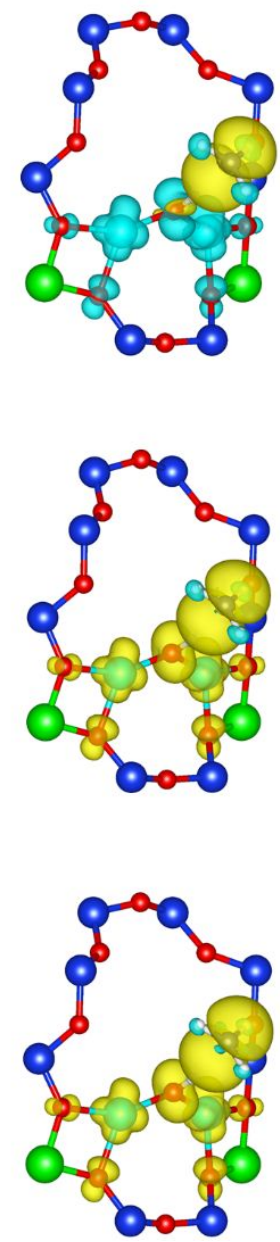

Figure S8. Visualization of atomic spin densities (yellow and blue isosurfaces correspond to positive and negative spin densities, respectively) and $\mathbf{C}-\mathbf{H}$ bond length for $\mathbf{R C}, \mathbf{T S}$, and $\mathbf{R I}$ of $\left[\mathrm{Cu}_{2}(\mu-\mathrm{O})\right]^{2+}$ ZSM-5 in the corresponding ground state. 

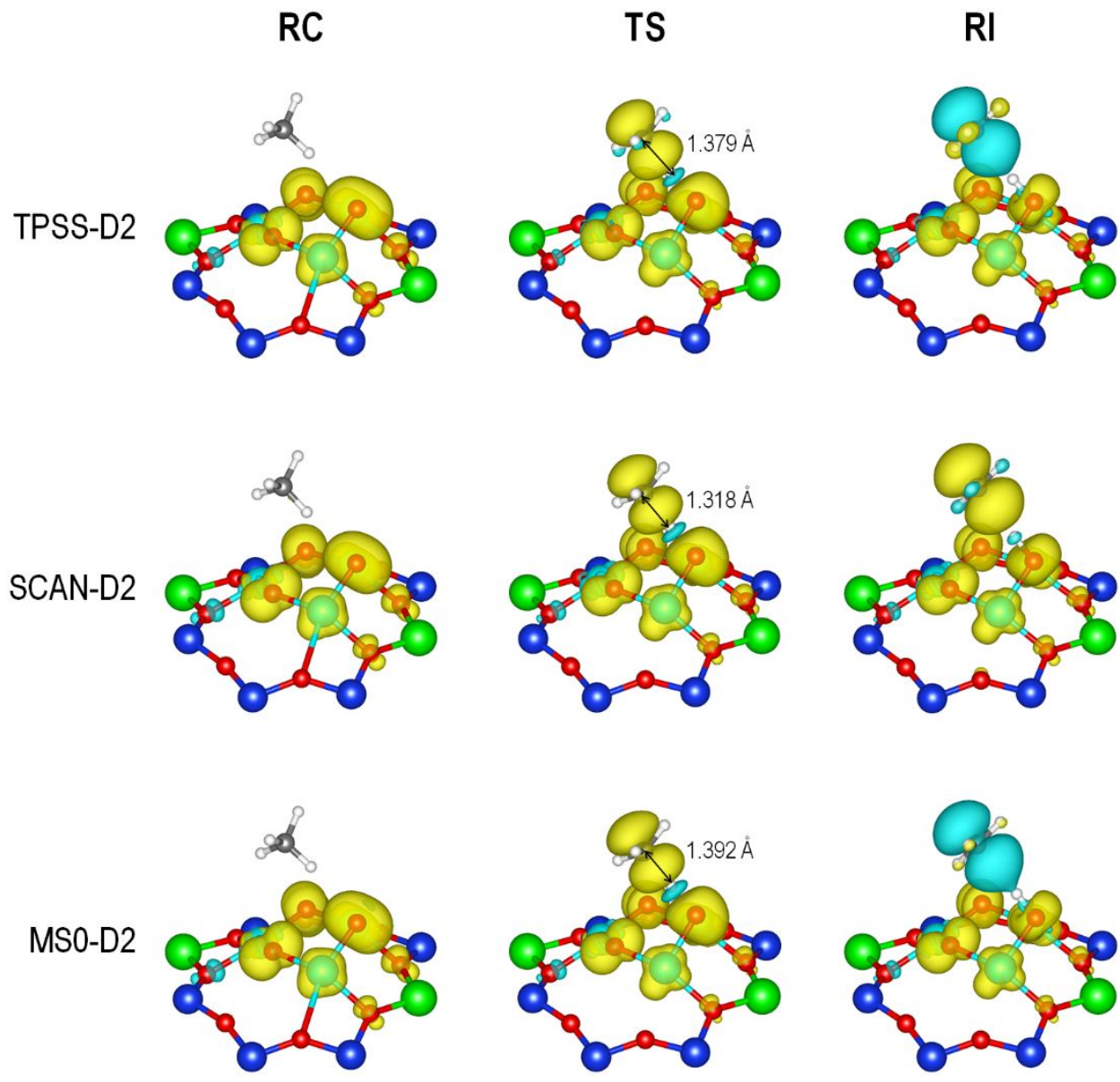

Figure S9. Visualization of atomic spin densities (yellow and blue isosurfaces correspond to positive and negative spin densities, respectively) and $\mathbf{C}-\mathrm{H}$ bond length for $\mathbf{R C}$, TS, and $\mathbf{R I}$ of $\left[\mathrm{Cu}_{3}(\mu-\mathrm{O})_{3}\right]^{2+}$ MOR in the corresponding ground state. 